\title{
Benchmarking the Performance of
} Exchange-Correlation Functionals for

\section{Predicting Two-Photon Absorption Strengths}

\author{
Maarten T. P. Beerepoot, ${ }^{\dagger}$ Md. Mehboob Alam,$^{\dagger}$ Joanna Bednarska ${ }^{\ddagger}$ Wojciech \\ Bartkowiak, ${ }^{\ddagger}$ Kenneth Ruud, ${ }^{*} \dagger$ and Robert Zaleśny ${ }^{*, \ddagger}$ \\ $\dagger$ Hylleraas Centre for Quantum Molecular Sciences, Department of Chemistry, UiT The \\ Arctic University of Norway, N-9037 Tromsø, Norway \\ $\ddagger$ Department of Physical and Quantum Chemistry, Faculty of Chemistry, Wrockaw \\ University of Science and Technology, Wyb. Wyspianskiego 27, PL-50370 Wrockaw, Poland \\ E-mail: kenneth.ruud@uit.no; robert.zalesny@pwr.edu.pl
}




\begin{abstract}
The present work aims at studying the performance of exchange-correlation functionals for the prediction of two-photon absorption (2PA) strengths. For this purpose, we considered six common functionals used for studying $2 \mathrm{PA}$ processes and tested these on six organoboron chelates. The set consisted of two semi-local (PBE and BLYP), two hybrid (B3LYP and PBE0), and two range-separated (LC-BLYP and CAM-B3LYP) functionals. The RI-CC2 method was chosen as the reference level and was found to give results consistent with the experimental data that are available for three of the molecules considered. Of the six exchange-correlation functionals studied, only the range-separated functionals predict an ordering of the $2 \mathrm{PA}$ strengths that is consistent with experiment and with RI-CC2 results. Even though the range-separated functionals predict correct relative trends, the absolute values for the 2PA strengths are underestimated by a factor of two to six for the molecules considered. An in-depth analysis, based on the derived generalized few-state model expression for the 2PA strength for a coupled-cluster wavefunction, reveals that the problem with these functionals can be linked to underestimated excited-state dipole moments and-to a lesser extentoverestimated excitation energies. The semi-local and hybrid functionals exhibit less predictable errors and a variation in the $2 \mathrm{PA}$ strengths in disagreement with the reference values. The semi-local and hybrid functionals show smaller average errors than the range-separated functionals, but our analysis reveals that this is due to fortuitous error cancellation between excitation energies and the transition dipole moments. Our results constitute a warning against using currently available exchange-correlation functionals in the prediction of $2 \mathrm{PA}$ strengths and highlight the need for functionals that correctly describe the electron density of excited electronic states.
\end{abstract}




\section{Introduction}

The two-photon absorption (2PA) process was predicted by Maria Göppert-Mayer in 1931, ${ }^{1}$ but the theory had to wait 30 years for experimental confirmation. ${ }^{2,3}$ Since then, one has witnessed a number of applications of this process. For instance, 2PA can be employed in the field of atomic and molecular spectroscopy to identify symmetry-forbidden transitions ${ }^{4}$ or to record Doppler-free spectra. ${ }^{5}$ Among the technology-related applications of 2PA are bioimaging ${ }^{6-9}$ and attempts to utilize the process for three-dimensional optical data storage. ${ }^{10,11}$ For some of these applications it is desirable to optimize the two-photon brightness, ${ }^{12,13}$ which is the product of the $2 \mathrm{PA}$ cross section and the fluorescence quantum yield. The simultaneous tuning of these parameters is by no means a trivial task. Despite difficulties, there has been a quest for the maximization of the $2 \mathrm{PA}$ cross section of molecules, largely relying on insights from theory. ${ }^{14-23}$ In fact, electronic structure calculations and related few-state model analyses frequently support experimental studies. ${ }^{14,24,25}$ The majority of computational studies are undertaken to understand the relation between chemical/electronic structure and 2PA activity and this has become an important ingredient in material design. However, the success of the predictions obtained by computer simulations depends heavily on the accuracy of the electronic structure methods used.

The first attempts at the quantum-chemical calculation of 2PA spectra of molecules were undertaken in the 1960's and 1970's, in most cases using semi-empirical Hamiltonians. ${ }^{4,26-29}$ Response theory formalisms were developed for various reference wave functions in the 1980's and 1990's and these were first implemented in the DALTON quantum chemistry program, ${ }^{30-34}$ thus allowing for fully ab-initio calculations of $2 \mathrm{PA}$ spectra. Nowadays, several other quantum-chemistry programs allow the calculation of $2 \mathrm{PA}$ spectra, including TURBOMOLE, ${ }^{35}$ GAMESS US ${ }^{36}$ and QCHEM. ${ }^{37}$ Thanks to efficient implementations, it is now possible to simulate electronic 2PA spectra of molecules composed of a few tens of atoms using coupled-cluster (CC) wavefunctions. Despite these recent advances, density functional theory (DFT) is still the most frequent choice for studying electronic structure and two- 
photon transition intensities for larger molecules. However, there are several striking reports regarding the poor and unsystematic performance of exchange-correlation functionals in this area. ${ }^{38,39}$ The $2 \mathrm{PA}$ cross section is proportional to the $2 \mathrm{PA}$ strength $\left(\delta_{0 J}^{2 \mathrm{PA}}\right)$ corresponding to a transition from the ground state $(0)$ to the final state $(J)$, and the product of the photon energies $\omega_{1} \omega_{2}$, which fulfill the resonance condition $\omega_{1}+\omega_{2}=\omega_{J}-\omega_{0}$. Reliable predictions of electronic 2PA spectra require that both excitation energies and 2PA strengths are accurately determined. It is now well recognized that many exchange-correlation functionals have difficulties in predicting the excitation energies to Rydberg and charge-transfer states. ${ }^{40-45}$ Considering that one of the paradigms in molecular nonlinear optics is the optimization of the chemical structures to maximize effective charge-transfer length, the use of DFT for these molecules can be troublesome. To overcome these difficulties, range-separated functionals were proposed. ${ }^{46,47}$ In fact, these functionals reduce average errors in excitation energies for Rydberg and charge-transfer excitations significantly. ${ }^{45}$ For this reason, the CAM-B3LYP functional gained some popularity in studies of $2 \mathrm{PA}$ spectra of extended $\pi$-conjugated systems. However, the CAM-B3LYP functional gives 2PA strengths that are underestimated in comparison with the reference $\mathrm{CC}$ values $^{38}$ even though it improves upon conventional functionals in predicting excitation energies to charge-transfer states. The subject in question becomes even more intriguing once the vibrational fine structure of bands in electronic 2PA spectra is considered. There are several reports demonstrating that conventional hybrid functionals, like B3LYP, yield disastrous predictions of geometric derivatives of second-order transition moments. ${ }^{48,49}$ From this perspective, it is a much safer strategy to employ rangeseparated functionals for simulations of vibronic structure in 2PA spectra.

All these results motivate the present study. The goal of this paper is to perform an in-depth analysis of the performance of several exchange-correlation functionals, including hybrid and range-separated hybrid functionals, in predicting electronic 2PA spectra. We will use CC2 results as a reference. An earlier benchmark work has shown that 2PA strengths predicted by the CC2 method are in very good agreement with more accurate CCSD results. ${ }^{38}$ 
The analysis will encompass not only electronic two-photon transition strengths but also electronic structure parameters like excitation energies and transition moments between electronic states. A convenient framework for such an analysis is the generalized few-state model, which allows essential intermediate states to be identified while at the same time taking into account the vectorial character of transition moments. ${ }^{50}$ The latter gives rise to the channel interference description of multiphoton absorption processes, for which the magnitude as well as the relative orientation of different transition dipole moments is crucial. At the CC level of theory, however, left and right transition moments may differ due to the non-Hermitian structure of the theory. ${ }^{35}$ In this work, in an attempt to pinpoint the differences between electronic structure parameters at the DFT and CC levels, we have taken the non-Hermitian structure of $\mathrm{CC}$ response theory into account and developed a generalized few-states model for CC wavefunctions.

For the in-depth assessment of the exchange-correlation functionals, we have chosen a series of six four-coordinate organoboron N,C chelates which have recently been studied experimentally (see Scheme 1). ${ }^{51}$

The remainder of this paper is organized as follows. A concise outline of the theory of the general few-state model for CC wavefunctions will be presented in Section 2. The computational details are presented in Section 3 and our results in Section 4. A summary of the main findings is presented in Section 5 .

\section{Theory}

In the case of one source of linearly polarized light and within the CC theory framework, the

rotationally averaged two-photon transition strength between states 0 and $J$ is given by: ${ }^{32,35}$

$$
\delta_{0 J}=\frac{1}{15} \sum_{\mu} \sum_{\nu}\left[M_{J \leftarrow 0}^{\mu \mu} M_{0 \leftarrow J}^{\nu \nu}+M_{J \leftarrow 0}^{\mu \nu} M_{0 \leftarrow J}^{\mu \nu}+M_{J \leftarrow 0}^{\mu \nu} M_{0 \leftarrow J}^{\nu \mu}\right] \quad \mu, \nu \in x, y, z
$$


where the symbols $M_{J \leftarrow 0}^{\mu \mu}$ and $M_{0 \leftarrow J}^{\nu \nu}$ are used to denote right and left second-order transition moments, respectively. In the case of one source of photons, i.e., $\omega=\frac{1}{2} \omega_{J}$, they read:

$$
\begin{aligned}
& M_{0 \leftarrow J}^{X Y}=\sum_{K}\left(\frac{\langle 0|X| K\rangle\langle K|Y| J\rangle}{\frac{1}{2} \omega_{J}-\omega_{K}}+\frac{\langle 0|Y| K\rangle\langle K|X| J\rangle}{\frac{1}{2} \omega_{J}-\omega_{K}}\right) \\
& M_{J \leftarrow 0}^{X Y}=\sum_{K}\left(\frac{\langle J|X| K\rangle\langle K|Y| 0\rangle}{\frac{1}{2} \omega_{J}-\omega_{K}}+\frac{\langle J|Y| K\rangle\langle K|X| 0\rangle}{\frac{1}{2} \omega_{J}-\omega_{K}}\right)
\end{aligned}
$$

Inserting Eqs. 2 and 3 into Eq. 1, one can derive the expression for a generalized few-state model for non-Hermitian theories, where the left and right transition moments are different. The derivation is presented in the Supporting Information. The final expression for the 2PA strength is given by:

$$
\begin{aligned}
\delta_{0 J K L}^{\mathrm{GFSM}} & =\sum_{K} \sum_{L} \frac{2}{15 \Delta E_{K} \Delta E_{L}}(\alpha+\beta), \\
\alpha & =\left|\mu^{J K}\right|\left|\mu^{K 0}\right|\left|\mu^{0 L}\right|\left|\mu^{L J}\right|\left(\cos \theta_{J K}^{K 0} \cos \theta_{0 L}^{L J}+\cos \theta_{J K}^{0 L} \cos \theta_{K 0}^{L J}+\cos \theta_{J K}^{L J} \cos \theta_{K 0}^{0 L}\right) \\
\beta & =\left|\mu^{J L}\right|\left|\mu^{L 0}\right|\left|\mu^{0 K}\right|\left|\mu^{K J}\right|\left(\cos \theta_{J L}^{L 0} \cos \theta_{0 K}^{K J}+\cos \theta_{J L}^{0 K} \cos \theta_{L 0}^{K J}+\cos \theta_{J L}^{K J} \cos \theta_{L 0}^{0 K}\right)
\end{aligned}
$$

In the above expression, the superscripts distinguish between right $(L 0)$ and left $(0 L)$ moments and $\Delta E_{K}=\frac{1}{2} \omega_{J}-\omega_{K}$. The term $\theta_{P Q}^{R S}$ in Eq. 4 represents the angle between the transition dipole moment vectors $\mu^{P Q}$ and $\mu^{R S}$. In the case of theories with Hermitian structure, i.e., where the left and right moments are equal, the above expression reduces to the one derived previously within the framework of time-dependent DFT: ${ }^{52}$

$$
\begin{aligned}
\delta_{0 J K L}^{\mathrm{GFSM}}= & \sum_{K} \sum_{L} \frac{4}{15 \Delta E_{K} \Delta E_{L}} \times \\
& \left|\mu^{0 K}\right|\left|\mu^{K J}\right|\left|\mu^{0 L}\right|\left|\mu^{L J}\right|\left(\cos \theta_{J K}^{0 K} \cos \theta_{0 L}^{L J}+\cos \theta_{J K}^{0 L} \cos \theta_{0 K}^{L J}+\cos \theta_{J K}^{L J} \cos \theta_{0 K}^{0 L}\right)
\end{aligned}
$$

Any number of intermediate states $K$ and $L$ can be chosen in the generalized few-state model expressions in Eqs. 4 and 5. In this work we will make use of a two-state model (2SM), in which $K$ and $L$ can be either the ground state 0 or the final excited state $J$. Four terms 
contribute to the 2SM expression for $\delta: \delta_{0 J 00}, \delta_{0 J 0 J}, \delta_{0 J J 0}$ and $\delta_{0 J J J}$, for which we will use the compact notations $\delta_{00}, \delta_{0 J}, \delta_{J 0}$ and $\delta_{J J}$, respectively.

\section{Computational details}

The geometries of all six compounds in Scheme 1 were optimized in the gas phase using the B3LYP functional ${ }^{53}$ and the cc-pVTZ basis set ${ }^{54}$ with the aid of the GAUSSIAN 09 program. ${ }^{55}$ All six compounds are neutral. The stationary points obtained were confirmed to be minima by evaluation of the Hessian. Gas-phase electronic structure calculations were performed at the optimized geometries to determine the poles and residues of the linear and quadratic response functions. The GAMESS US program was employed to determine the one- and two-photon absorption spectra based on DFT and the cc-pVDZ basis set. ${ }^{36}$ The palette of exchange-correlation functionals consisted of semi-local functionals (BLYP ${ }^{56,57}$ and $\mathrm{PBE}^{58}$ ), global hybrids (B3LYP 53 and PBE0 ${ }^{59,60}$ ) and range-separated hybrids (CAMB3LYP $^{46}$ and LC-BLYP ${ }^{47}$ ). The value of the range-separation parameter $\mu$ was set to 0.33 in the latter two functionals. In addition, RI-CC2 calculations were performed using the TURBOMOLE program. ${ }^{35,61}$ In these calculations, the cc-pVDZ basis set ${ }^{54}$ and the corresponding recommended auxiliary basis set ${ }^{62}$ were used to determine the electronic structure. The aug-cc-pVDZ basis set ${ }^{54}$ was employed to calculate the two-photon absorption strength for the first electronic excitation in molecules $\mathbf{1}$ and $\mathbf{2}$. The RI-CC2/cc-pVDZ results deviated by only $5 \%$ and $1 \%$, respectively, from the RI-CC2/aug-cc-pVDZ results. ${ }^{63}$ In the case of the CC2 method, left and right transition moments are different and in some cases even the sign of the moments is different, leading to negative values for oscillator strengths. For this reason, the sign differences in left and right transition moments were checked for all analyzed transitions to safeguard the correctness of the results obtained based on the generalized few-state models. In order to get further insight into the response theory results, the 2PA process has been further analyzed in terms of generalized few-state models at both 
the CC2 and DFT levels of theory. The scripts used for this analysis as well as the output from the scripts is available in an online repository. ${ }^{63}$ This repository also contains output from all calculations discussed in the text.

\section{Results and discussion}

We start this section with a discussion of the electronic structure of the four-coordinate organoboron N,C chelates. The excitation energies corresponding to the transitions to the two lowest singlet-excited states are shown in Table 1. Figure 1 shows the dominant oneelectron orbital transitions for the $\mathrm{S}_{0} \rightarrow \mathrm{S}_{1}$ and $\mathrm{S}_{0} \rightarrow \mathrm{S}_{2}$ electronic excitations. It is clear from the figure that the RI-CC2 method as well as the CAM-B3LYP, LC-BLYP and PBE0 functionals predict that the $\mathrm{S}_{0} \rightarrow \mathrm{S}_{1}$ and $\mathrm{S}_{0} \rightarrow \mathrm{S}_{2}$ excitations in all molecules (1-6) are dominated by the $\mathrm{HOMO} \rightarrow$ LUMO and HOMO-1 $\rightarrow$ LUMO transitions, respectively. This also holds for the B3LYP functional, except for molecule 5. The dominant orbital transitions in molecule 5 for B3LYP, BLYP and PBE are very different from the other methods and involve both HOMO-1 and HOMO-2 to LUMO transitions. For PBE and BLYP, the first two excited states in molecules $\mathbf{2}, \mathbf{4}$, and $\mathbf{6}$ are interchanged, whereas the dominating orbital character for molecules $\mathbf{1}$ and $\mathbf{3}$ are consistent with the range-separated functionals and the RI-CC2 method. In what follows, we will analyze only the electronic excitation dominated by the HOMO $\rightarrow$ LUMO transition. Thus, it is always $\mathrm{S}_{0} \rightarrow \mathrm{S}_{1}$ for RI-CC2 (1-6), CAMB3LYP (1-6), LC-BLYP (1-6) and B3LYP $(\mathbf{1}-\mathbf{4}, \mathbf{6})$, while in the case of BLYP and PBE it is either $\mathrm{S}_{0} \rightarrow \mathrm{S}_{1}(\mathbf{1}, \mathbf{3})$ or $\mathrm{S}_{0} \rightarrow \mathrm{S}_{2}(\mathbf{2}, \mathbf{4}, \mathbf{6})$. We will not analyze the electronic structure of molecule 5 for B3LYP, BLYP and PBE due to the complicated orbital character of the electronic excitations predicted by those functionals. For the sake of consistency, molecule 5 will be excluded from part of the comparative discussions.

Table 1 also contains the values of the 2PA strength corresponding to the two lowest singlet excited states for molecules 1-6, while Figure 2 shows the comparison of the two- 
photon transition strength, $\delta^{2 \mathrm{PA}}$ (lower inset) and 2PA cross section, $\sigma^{2 \mathrm{PA}}$ (upper inset) only for the electronic excitation dominated by the $\mathrm{HOMO} \rightarrow \mathrm{LUMO}$ transition for molecules 1-4 and 6. The upper inset in Figure 2 shows the comparison of calculated $2 \mathrm{PA}$ cross sections with experimental data available for molecules $\mathbf{1}, \mathbf{2}$ and $\mathbf{4}$. This quantity involves a product of $\delta^{2 \mathrm{PA}}$ and the square of the excitation energy. This comparison is made under two assumptions: firstly, the same value for the band width is assumed for all three molecules; secondly, we assume a similar solvent effect for all three molecules and compare theoretical values for molecules in the gas phase with experimental data obtained in the non-polar solvent chloroform $\left(\mathrm{CHCl}_{3}\right)$. In order to limit the impact of these two assumptions, the upper inset shows the comparison of relative $\sigma^{2 \mathrm{PA}}$ with the value for molecule $\mathbf{1}$ set to one. The experimental data (in black) shows roughly twofold and fourfold increase in $\sigma^{2 \mathrm{PA}}$ on passing from molecule 1 to $\mathbf{2}$ and from molecule 1 to $\mathbf{4}$, respectively. The best agreement with the experimental trend is observed for the RI-CC2 method, which gives support for using this method as reference for the assessment of two-photon transition strengths obtained using the exchange-correlation functionals. The range-separated functionals (CAM-B3LYP and LC-BLYP) also predict the order correctly, while the remaining four functionals give an incorrect trend. Indeed, $\sigma^{2 \mathrm{PA}}$ for molecule $\mathbf{2}$ is smaller than the value for molecule $\mathbf{1}$ for all four remaining functionals and the increase on passing from molecule $\mathbf{1}$ to molecule $\mathbf{4}$ is roughly 1.5 instead of the experimental factor of four.

The absolute 2PA strengths $\delta^{2 \mathrm{PA}}$ (lower panel of Figure 2) for the exchange-correlation functionals differ significantly from the RI-CC2 values. The range-separated functionals always predict the smallest values for $\delta^{2 \mathrm{PA}}$. In fact, the average ratio $\delta_{\mathrm{CC} 2}^{2 \mathrm{PA}} / \delta_{\mathrm{DFT}}^{2 \mathrm{PA}}$ is 3.28 and 4.08 for CAM-B3LYP and LC-BLYP (Table 2) with maximum values of 4.74 and 5.70, respectively. The underestimation of $\delta^{2 \mathrm{PA}}$ by CAM-B3LYP is similar to earlier work, in which a corresponding factor ranging from two to four was found for a different set of molecules. ${ }^{38}$ The average ratio $\delta_{\mathrm{CC} 2}^{2 \mathrm{PA}} / \delta_{\mathrm{DFT}}^{2 \mathrm{PA}}$ for the remaining functionals is much smaller and spans from 1.43 (BLYP) to 2.90 (PBE0). Thus, all functionals underestimate $\delta^{2 \mathrm{PA}}$ on average, compared 
to RI-CC2. In a few cases, $\delta^{2 \mathrm{PA}}$ is higher than for RI-CC2, with minimal values of the ratio $\delta_{\mathrm{CC} 2}^{2 \mathrm{PA}} / \delta_{\mathrm{DFT}}^{2 \mathrm{PA}}$ of $0.91(\mathrm{~B} 3 \mathrm{LYP}), 0.66(\mathrm{BLYP})$ and $0.71(\mathrm{PBE})$, all for molecule $\mathbf{1}$. The unsystematic performance of the exchange--correlation functionals is a striking result. One could argue that the deviation of the long-range corrected functionals is most predictable with less than a factor two difference between the minimum and maximum value for the ratio $\delta_{\mathrm{CC} 2}^{2 \mathrm{PA}} / \delta_{\mathrm{DFT}}^{2 \mathrm{PA}}$ among the six molecules (Table 2$)$. Values for $\delta_{\mathrm{CC} 2}^{2 \mathrm{PA}} / \delta_{\mathrm{DFT}}^{2 \mathrm{PA}}$ for all molecules are given in the Supporting Information.

In an attempt to shed light on the origin of the differences between $\delta^{2 \mathrm{PA}}$ predicted by the RI-CC2 method and by the various exchange-correlation functionals, we will take advantage of the generalized few-state model presented in Section 2. Although this model allows the use of an arbitrary number of intermediate excited states, for the sake of clarity we will base our analysis on the smallest reasonable number of states. Results based on a two-state model (i.e., only using the ground state 0 and final excited state $J$ ) are satisfactory while introducing more excited states only gives marginally better results. Figure 3 shows the comparison of $\delta^{2 \mathrm{PA}}$ from response theory $\left(\delta^{2 \mathrm{PA}}(\mathrm{RSP})\right)$ with the two-state model results. Results based on a two-state model are in most cases higher than those obtained with response theory, with a ratio between the two results well below a factor two. Note that these differences are much smaller than the relative differences in $\delta^{2 \mathrm{PA}}$ across the set of molecules studied. For the sake of completeness, Figure 3 also includes the comparison between orbital-relaxed (R) and orbital-unrelaxed (U) two-state model RI-CC2 results. ${ }^{64-66}$

To dig deeper into the nature of the $2 \mathrm{PA}$ process in the molecules studied, we will turn to the individual terms contributing to $\delta^{2 \mathrm{PA}}(2 \mathrm{SM})$, i.e., $\delta_{00}, \delta_{0 J}$ and $\delta_{J J}$. The final excited state $J$ is either $\mathrm{S}_{1}$ or $\mathrm{S}_{2}$, as discussed previously. Figure 4 contains the comparison of the three individual terms for all methods employed. Figure 4 provides several insights into the origin of the 2PA strengths and the differences between the methods employed. First, $\delta_{J J}$ dominates $\delta^{2 \mathrm{PA}}(2 \mathrm{SM})$ in most cases, while $\delta_{00}$ is similar to or larger than $\delta_{0 J}$. Thus, the correct prediction of $\delta_{J J}$ is crucial for a correct prediction of $\delta^{2 \mathrm{PA}}(2 \mathrm{SM})$ and, provided that the two- 
state model is a reliable approximation, also for the response-theory value of $\delta^{2 \mathrm{PA}}$. Indeed, $\delta_{J J}$ shows a similar trend to that predicted by response theory (and experiment) on passing from molecule 1 to 2 and from molecule 1 to 4 . Second, $\delta_{J J}$ is underestimated by the rangeseparated functionals CAM-B3LYP and LC-BLYP for all studied molecules, in comparison to RI-CC2. The other four functionals have a ratio $\delta_{J J}(\mathrm{CC} 2) / \delta_{J J}(\mathrm{DFT})$ that is either smaller or greater than one, depending on the molecule. Third, $\delta_{00}$ is underestimated by DFT with only one exception (molecule $\mathbf{1}$ for LC-BLYP). The long-range corrected functionals clearly come closest to the values of $\delta_{00}$ predicted by RI-CC2. Fourth, the $\delta_{0 J}(\mathrm{CC} 2) / \delta_{0 J}(\mathrm{DFT})$ ratio predicted using DFT can, similarly to the ratio for $\delta_{J J}$, be either smaller or greater than 1 . The range-separated functionals have negative values for $\delta_{0 J}$ for most molecules, in disagreement with the sign of $\delta_{0 J}$ for CC2. The sign of $\delta_{0 J}$ depends solely on the sign of the angle term for a two-state model (see Eq. 4 in Section 2), i.e., the relative orientation of different transition/dipole moment terms. The remaining four functionals predict a positive value for $\delta_{0 J}$ for all molecules, consistent with the RI-CC2 results. Based on these insights, one may point out two possible sources of the observed underestimated values of $\delta^{2 \mathrm{PA}}$ (see Figure 2) predicted by the range-separated functionals: i) the positive $\delta_{J J}$ term is largely underestimated (major source), ii) the $\delta_{0 J}$ term can be negative for some molecules, thus contributing to the discrepancies upon adding it to the the $\delta_{J J}$ term (minor source).

In order to gain further insight into the source of the observed discrepancies, the breakdown of the $\delta_{00}, \delta_{0 J}$ and $\delta_{J J}$ terms is shown in Figure 5 . To make a valid comparison between DFT and RI-CC2, the latter being a non-Hermitian theory, we show the products of left and right transition moments. For consistency, the other dipole terms are also presented as products. The lower inset in Figure 5 shows the "energy" term. ${ }^{67}$ According to Eq. 4, the $\delta_{00}$ and $\delta_{J J}$ terms are products of three out of four terms shown in Figure 5 multiplied by the angular term (not shown). We can thus pinpoint the sources of the discrepancy in $\delta_{00}$ and $\delta_{J J}$. We highlight the key observations from the analysis of Figure 5 separately for the range-separated functionals and the remaining four functionals. First, the range-separated 
functionals are closest to the reference method RI-CC2 for all contributions. Indeed, the range-separated functionals predict values of $\left|\mu_{00}\right|^{2}$ very similar to the values of the RI-CC2 method. In addition, these two functionals outperform the other four functionals in the calculation of $\left|\mu_{0 J}\right|\left|\mu_{J 0}\right|$. The same holds for the "energy" term (lower panel), which is however slightly underestimated by the range-separated functionals for all molecules, which is to be expected as they overestimate the excitation energy. ${ }^{45}$ The range-separated functionals significantly underestimate $\left|\mu_{J J}\right|^{2}$ for all molecules except molecule $\mathbf{3}$. The dominating $\delta_{J J}$ term is thus underestimated by the range-separated functionals due to too small values of $\left|\mu_{J J}\right|^{2}$ (major source) and an underestimated value of the "energy" term (minor source). In other words, the range-separated functionals largely underestimate the excited-state dipole moment of the final state in addition to a small overestimation of the excitation energy to that state. Second, the other four functionals also perform well for $\left|\mu_{00}\right|^{2}$, but do not follow any systematic trend across the set of studied molecules for the other terms. For example, the $\left|\mu_{J J}\right|^{2}$ term is either largely overestimated (molecules $\mathbf{1}$ and $\mathbf{3}$ ) or underestimated (molecules 4 and 6). Moreover, the $\left|\mu_{0 J}\right|\left|\mu_{J 0}\right|$ term is underestimated for the B3LYP, BLYP, PBE and PBE0 functionals, but to a very different degree for the different molecules. We observe a dramatic error cancellation between the "energy" term and the $\left|\mu_{0 J}\right|\left|\mu_{J 0}\right|$ term for those four functionals. The energy term is largely overestimated, while $\left|\mu_{0 J}\right|\left|\mu_{J 0}\right|$ is underestimated in an unsystematic manner. Since the product of $\left|\mu_{0 J}\right|\left|\mu_{J 0}\right|$ and the energy term appears in $\delta_{00}, \delta_{0 J}$ and $\delta_{J J}$, the success of a given functional in predicting $\delta^{2 \mathrm{PA}}$ depends to a very large extent on this error cancellation for a specific molecule. One should be aware of the unpredictable error in $\delta^{2 \mathrm{PA}}$ in general and this error cancellation in particular when claiming that hybrid functionals are to be preferred in calculations of $\delta^{2 \mathrm{PA}} .{ }^{68}$ 


\section{$5 \quad$ Summary and conclusions}

In summary, we have studied the performance of six density functionals (CAM-B3LYP, LCBLYP, B3LYP, PBE0, BLYP, and PBE) in predicting the 2PA strength of six organoboron N,C chelates with RI-CC2 as reference. For this purpose, we have also derived the generalized few-state model expression for 2PA strength for a $\mathrm{CC}$ wavefunction. Our analysis has revealed that $2 \mathrm{PA}$ strengths predicted by range-separated functionals are seriously underestimated, but in a relatively systematic manner. Errors for semi-local and hybrid functionals, on the other hand, are smaller on average but more unsystematic and system dependent. Hence, only range-separated functionals provide relative 2PA strengths in agreement with the theoretical reference and with experimental data for the investigated molecules. Our two-state model analysis has revealed that the smaller average error of semi-local and hybrid functionals is due to an error compensation between energy and transition dipole moment terms. Our work clearly demonstrates the need to develop functionals that can describe the electron density of excited electronic states, and not only the excitation energies and transition moments to these excited states.

\section{Acknowledgement}

This work was partially supported by the Research Council of Norway through its Centres of Excellence scheme (Grant number 262695) and through a FRIPRO grant (Grant number 250743). R.Z. acknowledges financial support from the Polish National Science Centre (Grant No. DEC-2013/10/A/ST4/00114). This work was also supported by the statutory activity subsidy from the Polish Ministry of Science and Higher Education for the Faculty of Chemistry of Wroclaw University of Science and Technology. The calculations were per-

formed in part at the Wroclaw Center for Networking and Supercomputing. The authors also acknowledge the financial support for action "LIMA" from The National Centre for Research and Development - Programme Operator of the Polish-Norwegian Research Programme. 


\section{Supporting Information Available}

Statistical data analysis for $\delta^{2 \mathrm{PA}}$ : values for $\xi$ for all molecules. Derivation of the general few-state model for $\mathrm{CC}$ wavefunctions. This material is available free of charge via the Internet at http://pubs . acs .org/.

\section{References}

(1) M. Göppert-Mayer, Ann. Phys. 1931, 9, 273-294.

(2) Peticolas, W.; Goldsborough, J.; Rieckhoff, K. Phys. Rev. Lett. 1963, 10, 43-45.

(3) Peticolas, W.; Rieckhoff, K. J. Chem. Phys. 1963, 39, 1347-1348.

(4) Birge, R. R.; Bennett, J. A.; Pierce, B. M.; Thomas, T. M. J. Am. Chem. Soc. 1978, 100, 1533-1539.

(5) Neusser, H. J.; Schlag, E. W. Angew. Chem. Int. Ed. Engl. 1992, 31, 263-273.

(6) Drobizhev, M.; Makarov, N. S.; Tillo, S. E.; Hughes, T. E.; Rebane, A. Nature Methods 2011, 8, 393-399.

(7) Streets, A. M.; Li, A.; Chen, T.; Huang, Y. Anal. Chem. 2014, 86, 8506-8513.

(8) Wang, B.-G.; König, K.; Halbhuber, K.-J. J. Microsc. 2010, 238, 1-20.

(9) Denk, W.; Strickler, J.; Webb, W. Science 1990, 248, 73-76.

(10) Parthenopoulos, D. A.; Rentzepis, P. M. Science 1989, 245, 843-845.

(11) Dvornikov, A. S.; Walker, E. P.; Rentzepis, P. M. J. Phys. Chem. A 2009, 113, 1363313644 .

(12) Pawlicki, M.; Collins, H. A.; Denning, R. G.; Anderson, H. L. Angew. Chem. Int. Ed. 2009, 48, 3244-3266. 
(13) Bednarska, J.; Zaleśny, R.; Wielgus, M.; Jędrzejewska, B.; Puttreddy, R.; Rissanen, K.; Bartkowiak, W.; Ågren, H.; Ośmiałowski, B. Phys. Chem. Chem. Phys. 2017, 19, $5705-5708$.

(14) Albota, M.; Beljonne, D.; Brédas, J.-L.; Ehrlich, J.; Fu, J.-Y.; Heikal, A.; Hess, S.; Kogej, T.; Levin, M.; Marder, S.; McCord-Maughon, D.; Perry, J.; Röckel, H.; Rumi, M. Science 1998, 281, 1653-1656.

(15) Zaleśny, R.; Bartkowiak, W.; Styrcz, S.; Leszczynski, J. J. Phys. Chem. A 2002, 106, $4032-4037$.

(16) Bartkowiak, W.; Zaleśny, R.; Leszczynski, J. Chem. Phys. 2003, 287, 103-112.

(17) Pati, S.; Marks, T.; Ratner, M. J. Am. Chem. Soc. 2001, 123, 7287-7291.

(18) Kogej, T.; Beljonne, D.; Meyers, F.; Perry, J.; Marder, S.; Brédas, J. Chem. Phys. Lett. 1998, 298, 1-6.

(19) Macak, P.; Luo, Y.; Norman, P.; Agren, H. J. Chem. Phys. 2000, 113, 7055-7061.

(20) Norman, P.; Cronstrand, P.; Ericsson, J. Chem. Phys. 2002, 285, 207-220.

(21) Luo, Y.; Norman, P.; Macak, P.; Ågren, H. J. Phys. Chem. A 2000, 104, 4718-4722.

(22) Norman, P.; Luo, Y.; Ågren, H. J. Chem. Phys. 1999, 111, 7758-7765.

(23) Lee, W.-H.; Lee, H.; Kim, J.-A.; Choi, J.-H.; Cho, M.; Jeon, S.-J.; Cho, B. J. Am. Chem. Soc. 2001, 123, 10658-10667.

(24) Wielgus, M.; Zaleśny, R.; Murugan, N. A.; Kongsted, J.; Ågren, H.; Samoc, M.; Bartkowiak, W. ChemPhysChem 2013, 14, 3731-3739.

(25) Olesiak-Banska, J.; Matczyszyn, K.; Zaleśny, R.; Murugan, N. A.; Kongsted, J.; Ågren, H.; Bartkowiak, W.; Samoc, M. J. Phys. Chem. B 2013, 117, 12013-12019. 
(26) Honig, B.; Jortner, J.; Szöke, A. J. Chem. Phys. 1967, 46, 2714-2727.

(27) Honig, B.; Jortner, J. J. Chem. Phys. 1967, 47, 3698-3703.

(28) Birge, R.; Pierce, B. J. Chem. Phys. 1979, 70, 165-178.

(29) Marchese, F.; Seliskar, C.; Jaffé, H. J. Chem. Phys. 1980, 72, 4149-4203.

(30) Olsen, J.; Jørgensen, P. J. Chem. Phys. 1985, 82, 3235-3264.

(31) Sundholm, D.; Rizzo, A.; Jørgensen, P. J. Chem. Phys. 1994, 101, 4931-4935.

(32) Hättig, C.; Christiansen, O.; Jørgensen, P. J. Chem. Phys. 1998, 108, 8331-8354.

(33) Aidas, K.; Angeli, C.; Bak, K. L.; Bakken, V.; Bast, R.; Boman, L.; Christiansen, O.; Cimiraglia, R.; Coriani, S.; Dahle, P.; Dalskov, E. K.; Ekström, U.; Enevoldsen, T.; Eriksen, J. J.; Ettenhuber, P.; Fernández, B.; Ferrighi, L.; Fliegl, H.; Frediani, L.; Hald, K.; Halkier, A.; Hättig, C.; Heiberg, H.; Helgaker, T.; Hennum, A. C.; Hettema, H.; Hjertenæs, E.; Høst, S.; Høyvik, I.-M.; Iozzi, M. F.; Jansík, B.; Jensen, H. J. A.; Jonsson, D.; Jørgensen, P.; Kauczor, J.; Kirpekar, S.; Kjærgaard, T.; Klopper, W.; Knecht, S.; Kobayashi, R.; Koch, H.; Kongsted, J.; Krapp, A.; Kristensen, K.; Ligabue, A.; Lutnis, O. B.; Melo, J. I.; Mikkelsen, K. V.; Myhre, R. H.; Neiss, C.; Nielsen, C. B.; Norman, P.; Olsen, J.; Olsen, J. M. H.; Osted, A.; Packer, M. J.; Pawlowski, F.; Pedersen, T. B.; Provasi, P. F.; Reine, S.; Rinkevicius, Z.; Ruden, T. A.; Ruud, K.; Rybkin, V. V.; Sałek, P.; Samson, C. C. M.; de Meras, A. S.; Saue, T.; Sauer, S. P. A.; Schimmelpfennig, B.; Sneskov, K.; Steindal, A. H.; SylvesterHvid, K. O.; Taylor, P. R.; Teale, A. M.; Tellgren, E. I.; Tew, D. P.; Thorvaldsen, A. J.; Thøgersen, L.; Vahtras, O.; Watson, M. A.; Wilson, D. J. D.; Ziolkowski, M.; Ågren, H. WIREs Comput. Mol. Sci. 2014, 4, 269-284.

(34) Sałek, P.; Vahtras, O.; Guo, J.; Luo, Y.; Helgaker, T.; Ågren, H. Chem. Phys. Lett. 2003, 374, 446-452. 
(35) Friese, D. H.; Hättig, C.; Ruud, K. Phys. Chem. Chem. Phys. 2012, 14, 1175-1184.

(36) Zahariev, F.; Gordon, M. S. J. Chem. Phys. 2014, 140, 18A523.

(37) Nanda, K. D.; Krylov, A. I. J. Chem. Phys. 2015, 142, 064118.

(38) Beerepoot, M. T. P.; Friese, D. H.; List, N. H.; Kongsted, J.; Ruud, K. Phys. Chem. Chem. Phys. 2015, 17, 19306-19314.

(39) Zaleśny, R.; Murugan, N. A.; Tian, G.; Medved', M.; Ågren, H. J. Phys. Chem. B 2016, 120, 2323-2332.

(40) Dreuw, A.; Head-Gordon, M. Chem. Rev. 2005, 105, 4009.

(41) Jacquemin, D.; Wathelet, V.; Perpéte, E. A.; Adamo, C. J. Chem. Theory Comput. 2009, 5, 2420-2435.

(42) Laurent, A.; Jacquemin, D. Int. J. Quant. Chem. 2013, 113, 2019-2039.

(43) Guennic, B. L.; Jacquemin, D. Acc. Chem. Res. 2015, 48, 530-537.

(44) Charaf-Eddin, A.; Planchat, A.; Mennucci, B.; Adamo, C.; Jacquemin, D. J. Chem. Theory Comput. 2013, 9, 2749-2760.

(45) Peach, M. J. G.; Benfield, P.; Helgaker, T.; Tozer, D. J. J. Chem. Phys. 2008, 128, 044118 .

(46) Yanai, T.; Tew, D. P.; Handy, N. C. Chem. Phys. Lett. 2004, 393, $51-57$.

(47) Iikura, H.; Tsuneda, T.; Yanai, T.; Hirao, K. J. Chem. Phys. 2001, 115, 3540-3544.

(48) Bednarska, J.; Zaleśny, R.; Tian, G.; Murugan, N. A.; Ågren, H.; Bartkowiak, W. Molecules 2017, 22, 1643.

(49) Zaleśny, R.; Tian, G.; Hättig, C.; Bartkowiak, W.; Ågren, H. J. Comput. Chem. 2015, 36, 1124-1131. 
(50) Alam, M. M.; Chattopadhyaya, M.; Chakrabarti, S.; Ruud, K. Acc. Chem. Res. 2014, 47, 1604-1612.

(51) Pais, V. F.; Alcaide, M. M.; López-Rodríguez, R.; Collado, D.; Nájera, F.; PérezInestrosa, E.; Álvarez, E.; Lassaletta, J. M.; Fernández, R.; Ros, A.; Pischel, U. Chem. Eur. J 2015, 21, 15369-15376.

(52) Alam, M. M.; Chattopadhyaya, M.; Chakrabarti, S. Phys. Chem. Chem. Phys. 2012, 14, 1156-1165.

(53) Becke, A. D. J. Chem. Phys. 1993, 98, 5648-5652.

(54) Dunning Jr., T. H. J. Chem. Phys. 1989, 90, 1007-1023.

(55) Frisch, M. J.; Trucks, G. W.; Schlegel, H. B.; Scuseria, G. E.; Robb, M. A.; Cheeseman, J. R.; Scalmani, G.; Barone, V.; Mennucci, B.; Petersson, G. A.; Nakatsuji, H.; Caricato, M.; Li, X.; Hratchian, H. P.; Izmaylov, A. F.; Bloino, J.; Zheng, G.; Sonnenberg, J. L.; Hada, M.; Ehara, M.; Toyota, K.; Fukuda, R.; Hasegawa, J.; Ishida, M.; Nakajima, T.; Honda, Y.; Kitao, O.; Nakai, H.; Vreven, T.; Montgomery, J. A., Jr.; Peralta, J. E.; Ogliaro, F.; Bearpark, M.; Heyd, J. J.; Brothers, E.; Kudin, K. N.; Staroverov, V. N.; Kobayashi, R.; Normand, J.; Raghavachari, K.; Rendell, A.; Burant, J. C.; Iyengar, S. S.; Tomasi, J.; Cossi, M.; Rega, N.; Millam, J. M.; Klene, M.; Knox, J. E.; Cross, J. B.; Bakken, V.; Adamo, C.; Jaramillo, J.; Gomperts, R.; Stratmann, R. E.; Yazyev, O.; Austin, A. J.; Cammi, R.; Pomelli, C.; Ochterski, J. W.; Martin, R. L.; Morokuma, K.; Zakrzewski, V. G.; Voth, G. A.; Salvador, P.; Dannenberg, J. J.; Dapprich, S.; Daniels, A. D.; Farkas, Ö.; Foresman, J. B.; Ortiz, J. V.; Cioslowski, J.; Fox, D. J. Gaussian 2009. Gaussian Inc. Wallingford CT 2009.

(56) Becke, A. D. Phys. Rev. A 1988, 38, 3098-3100.

(57) Lee, C.; Yang, W.; Parr, R. G. Phys. Rev. B 1988, 37, 785-789. 
(58) Perdew, J. P.; Ernzerhof, M.; Burke, K. J. Chem. Phys. 1996, 105, 9982-9985.

(59) Adamo, C.; Barone, V. J. Chem. Phys. 1999, 110, 6158-6170.

(60) Ernzerhof, M.; Scuseria, G. E. J. Chem. Phys. 1999, 110, 5029-5036.

(61) TURBOMOLE V7.0 2015, a development of University of Karlsruhe and Forschungszentrum Karlsruhe GmbH, 1989-2007, TURBOMOLE GmbH, since 2007; available from http://www.turbomole.com (Last accessed 01 Apr. 17).

(62) Weigend, F.; Köhn, A.; Hättig, C. J. Chem. Phys. 2002, 116, 3175-3183.

(63) Beerepoot, M. T. P.; Alam, M. M.; Bednarska, J.; Bartkowiak, W.; Ruud, K.; Zalesny, R. Repository with calculation output files for this work. 2018; DOI: 10.6084/m9.figshare.5908921.v1.

(64) Christiansen, O.; Koch, H.; Jørgensen, P. Chem. Phys. Lett. 1995, 243, 409-418.

(65) Pawłowski, F.; Jørgensen, P.; Hättig, C. Chem. Phys. Lett. 2005, 413, 272-279.

(66) Helgaker, T.; Coriani, S.; Jørgensen, P.; Kristensen, K.; Olsen, J.; Ruud, K. Chem. Rev. 2012, 112, 543-631.

(67) Alam, M. M.; Beerepoot, M. T. P.; Ruud, K. J. Chem. Phys. 2017, 146, 244116.

(68) Salem, M. A.; Brown, A. J. Chem. Theory Comput. 2014, 10, 3260-3269. 

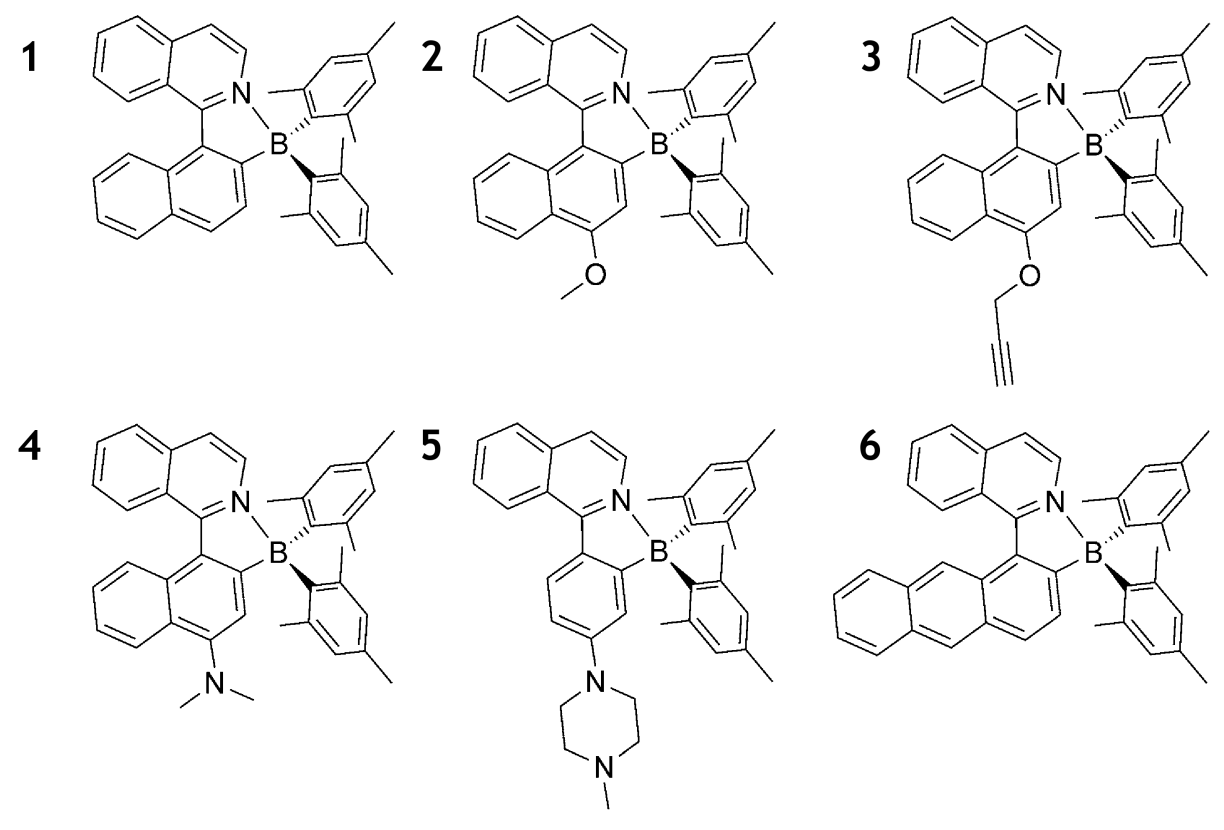

Scheme 1: The structures of four-coordinate organoboron N,C chelates ${ }^{51}$ studied in the present work. Reused with permission. (c) 2015 Wiley-VCH Verlag GmbH \& Co. KGaA, Weinheim. 


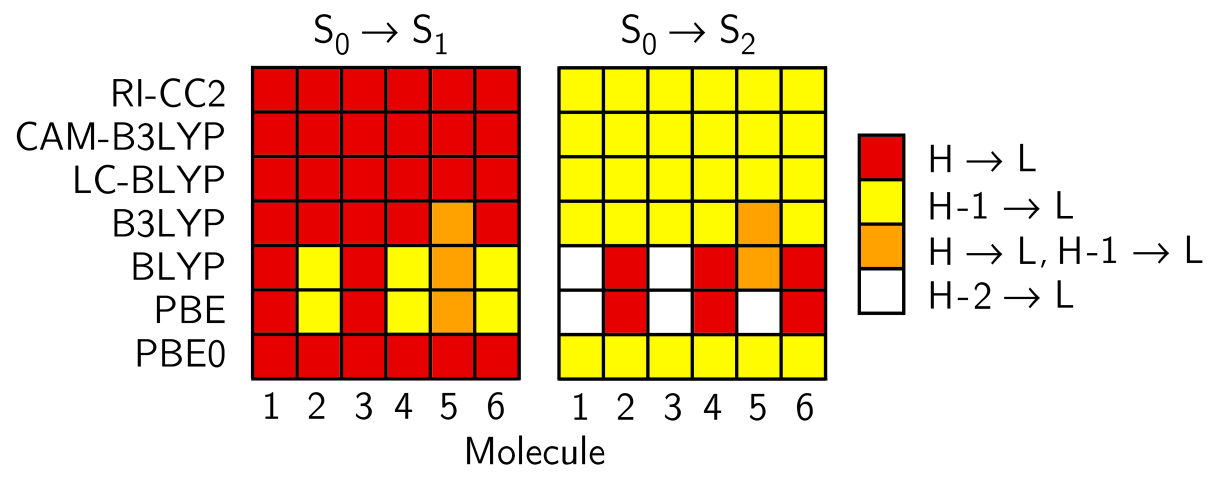

Figure 1: Dominating orbital character for the $\mathrm{S}_{0} \rightarrow \mathrm{S}_{1}$ (left) and $\mathrm{S}_{0} \rightarrow \mathrm{S}_{2}$ (right) electronic transitions. $\mathrm{H}=\mathrm{HOMO} ; \mathrm{L}=\mathrm{LUMO}$. 


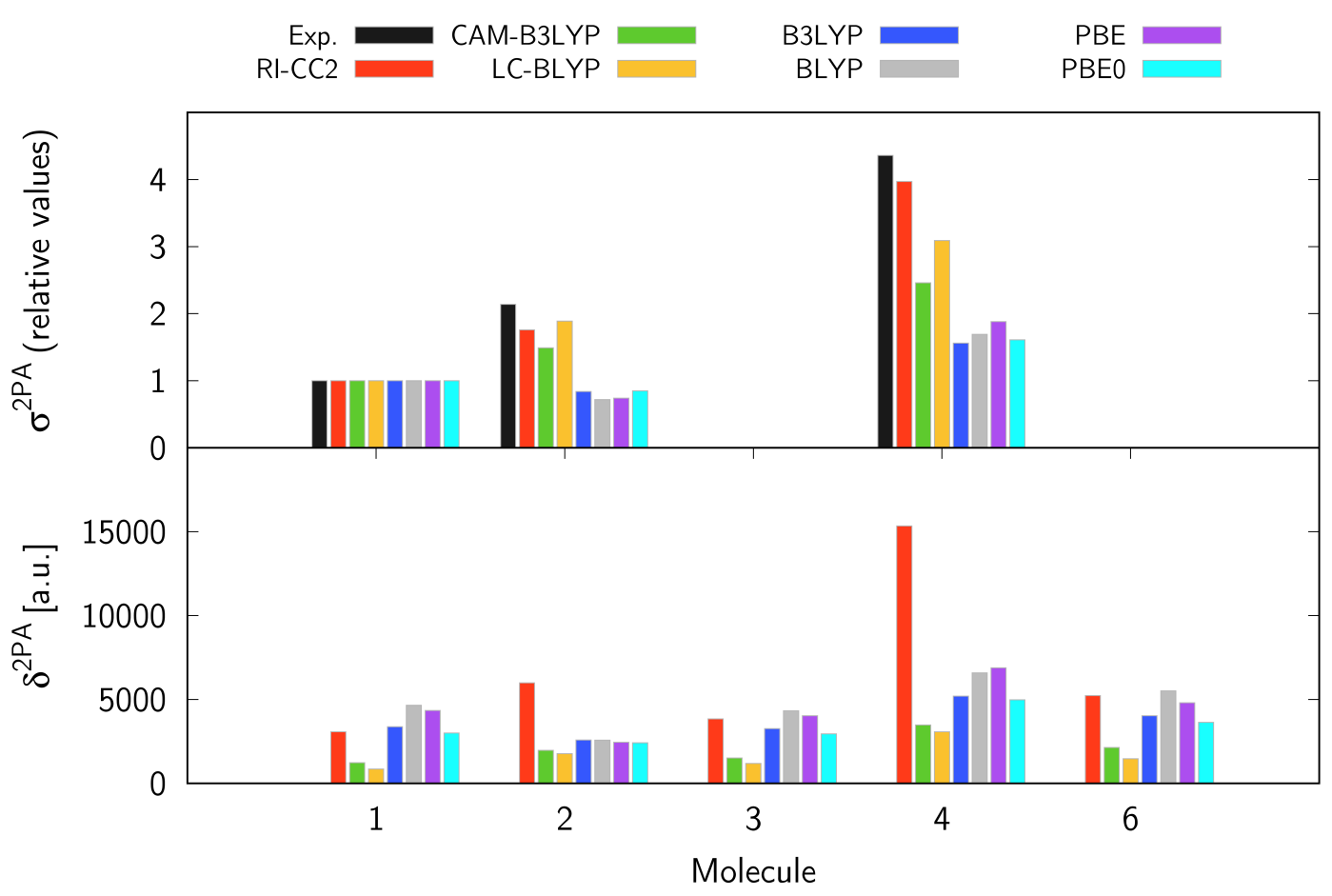

Figure 2: Relative two-photon absorption cross section $\sigma^{2 \mathrm{PA}}$ (upper panel) and calculated absolute two-photon transition strength $\delta^{2 \mathrm{PA}}$ (lower panel) corresponding to the lowest-energy electronic excitation dominated by the HOMO $\rightarrow$ LUMO transition $\left(\mathrm{S}_{0} \rightarrow \mathrm{S}_{1}\right.$ or $\mathrm{S}_{0} \rightarrow \mathrm{S}_{2}$, see Figure 1). $\sigma^{2 \mathrm{PA}}$ is only given for molecules where experimental data is available and is given relative to molecule $\mathbf{1}$. 


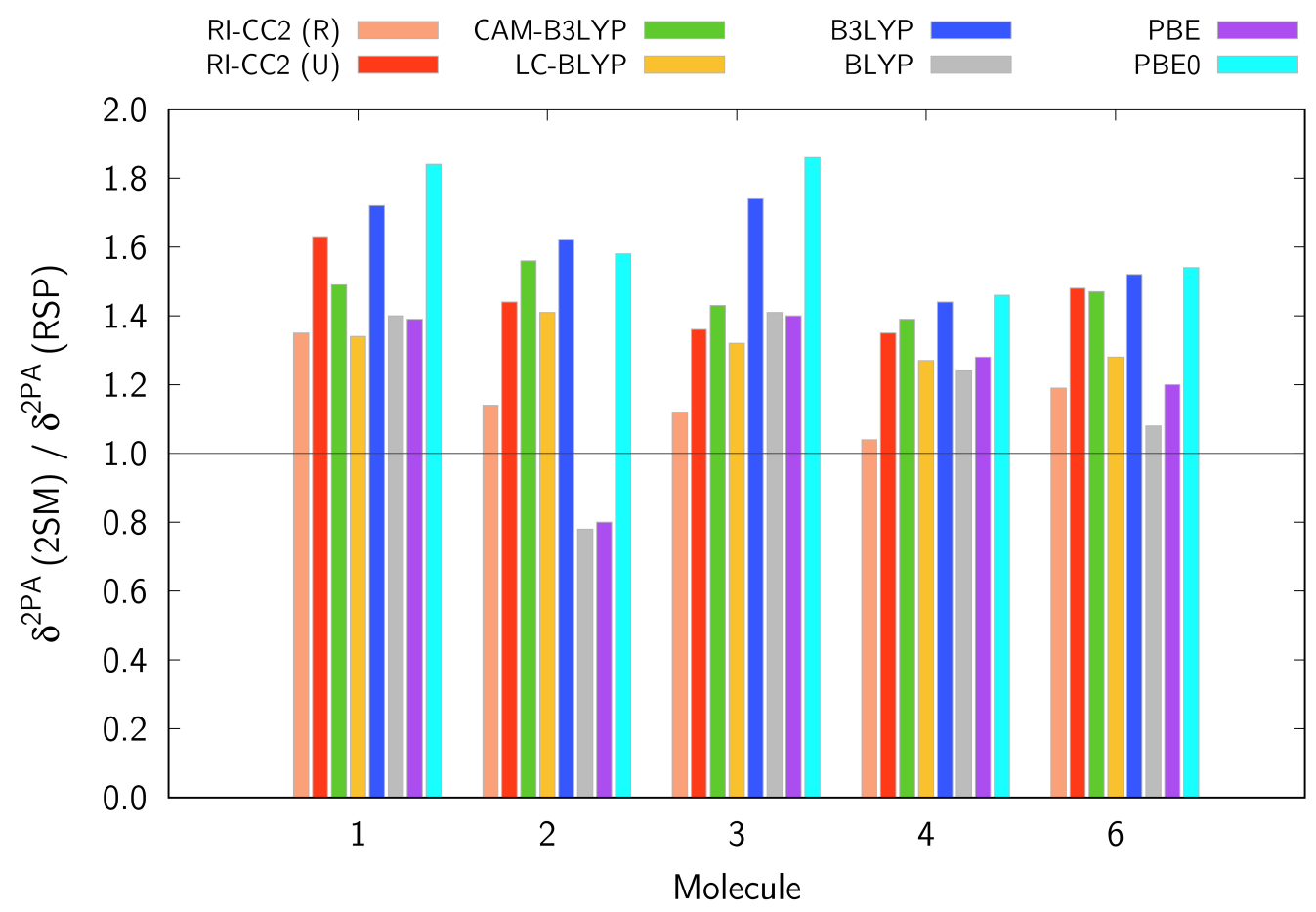

Figure 3: Ratio of the two-photon absorption strength $\delta^{2 \mathrm{PA}}$ computed with a two-state model (2SM) and the two-photon transition strengths computed with response theory (RSP) for the lowest-energy electronic excitation dominated by the HOMO $\rightarrow$ LUMO transition $\left(\mathrm{S}_{0} \rightarrow \mathrm{S}_{1}\right.$ or $\mathrm{S}_{0} \rightarrow \mathrm{S}_{2}$, see Figure 1). 

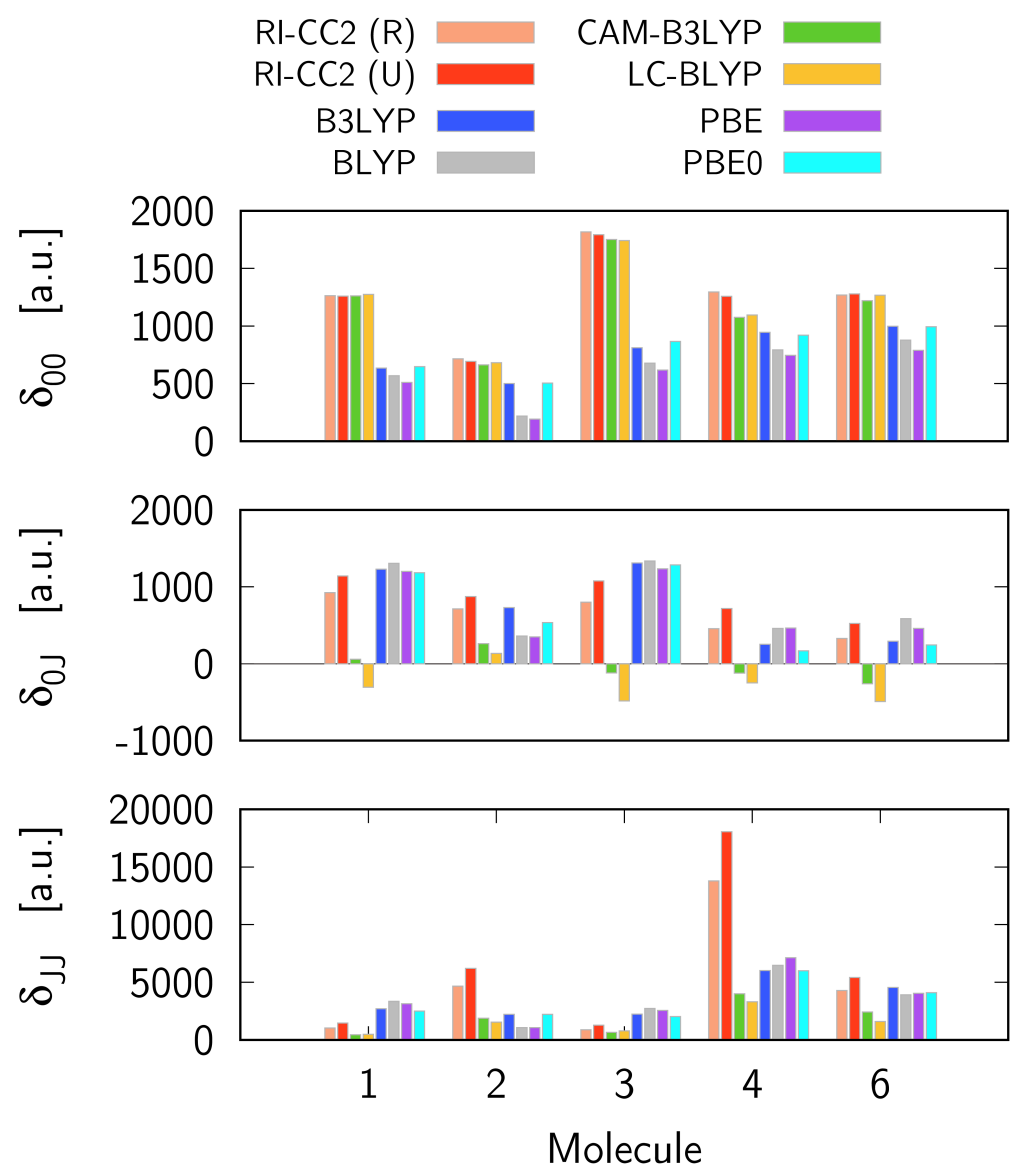

Figure 4: Terms contributing to the two-photon absorption strength $\delta$ within a two-state approximation for the lowest-energy electronic excitation dominated by the HOMO $\rightarrow$ LUMO transition $\left(\mathrm{S}_{0} \rightarrow \mathrm{S}_{1}\right.$ or $\mathrm{S}_{0} \rightarrow \mathrm{S}_{2}$, see Figure 1). 

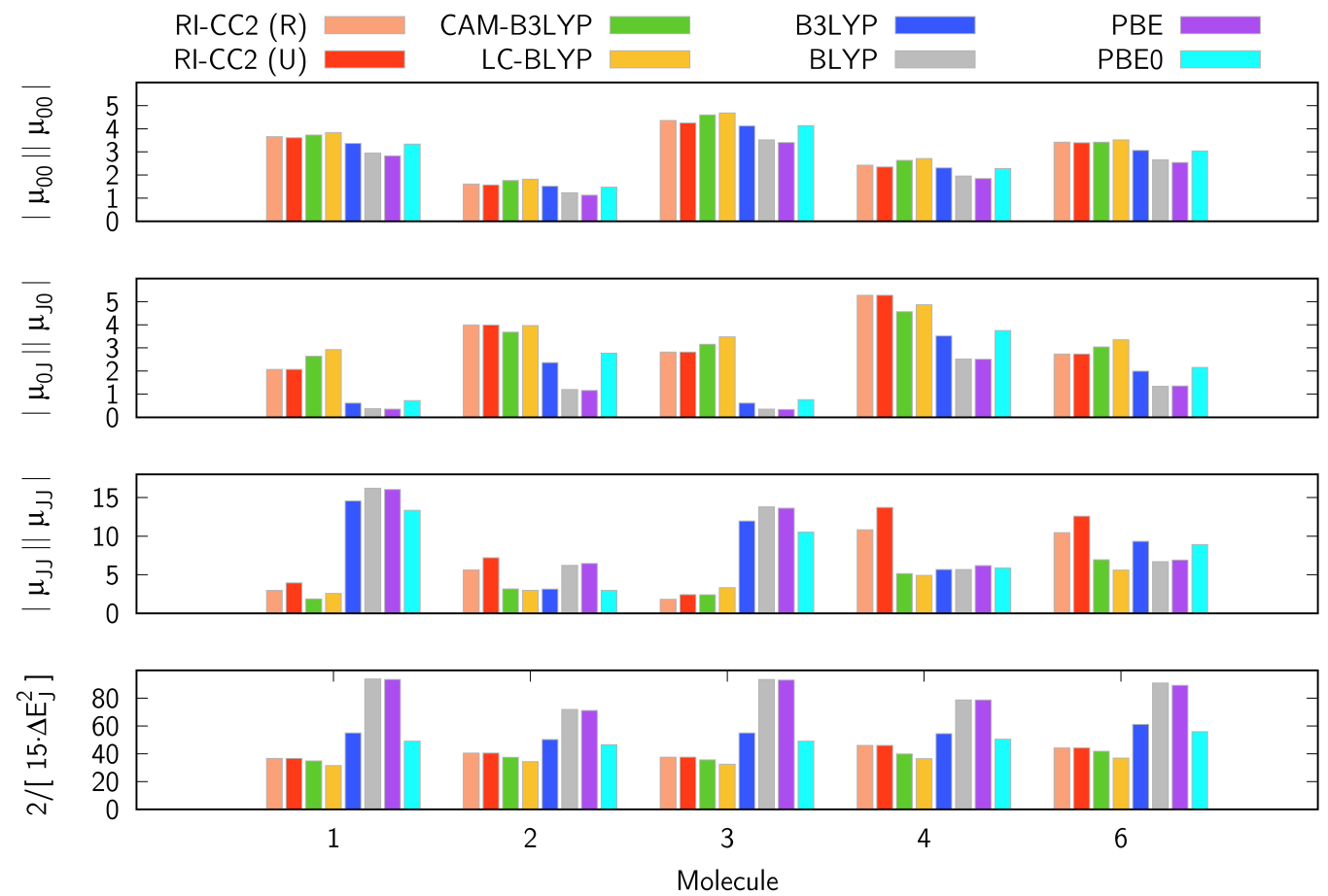

Figure 5: Dipole and energy terms contributing to the two-photon absorption strength within a two-state approximation for the lowest-energy electronic excitation dominated by the $\mathrm{HOMO} \rightarrow$ LUMO transition $\left(\mathrm{S}_{0} \rightarrow \mathrm{S}_{1}\right.$ or $\mathrm{S}_{0} \rightarrow \mathrm{S}_{2}$, see Figure 1$)$. 
Table 1: Excitation energies $(\Delta E$, in $\mathrm{eV})$ and two-photon transition strengths $\left(\delta^{2 \mathrm{PA}}\right.$, in a.u.) corresponding to excitations to the two lowest-energy singlet excited states.

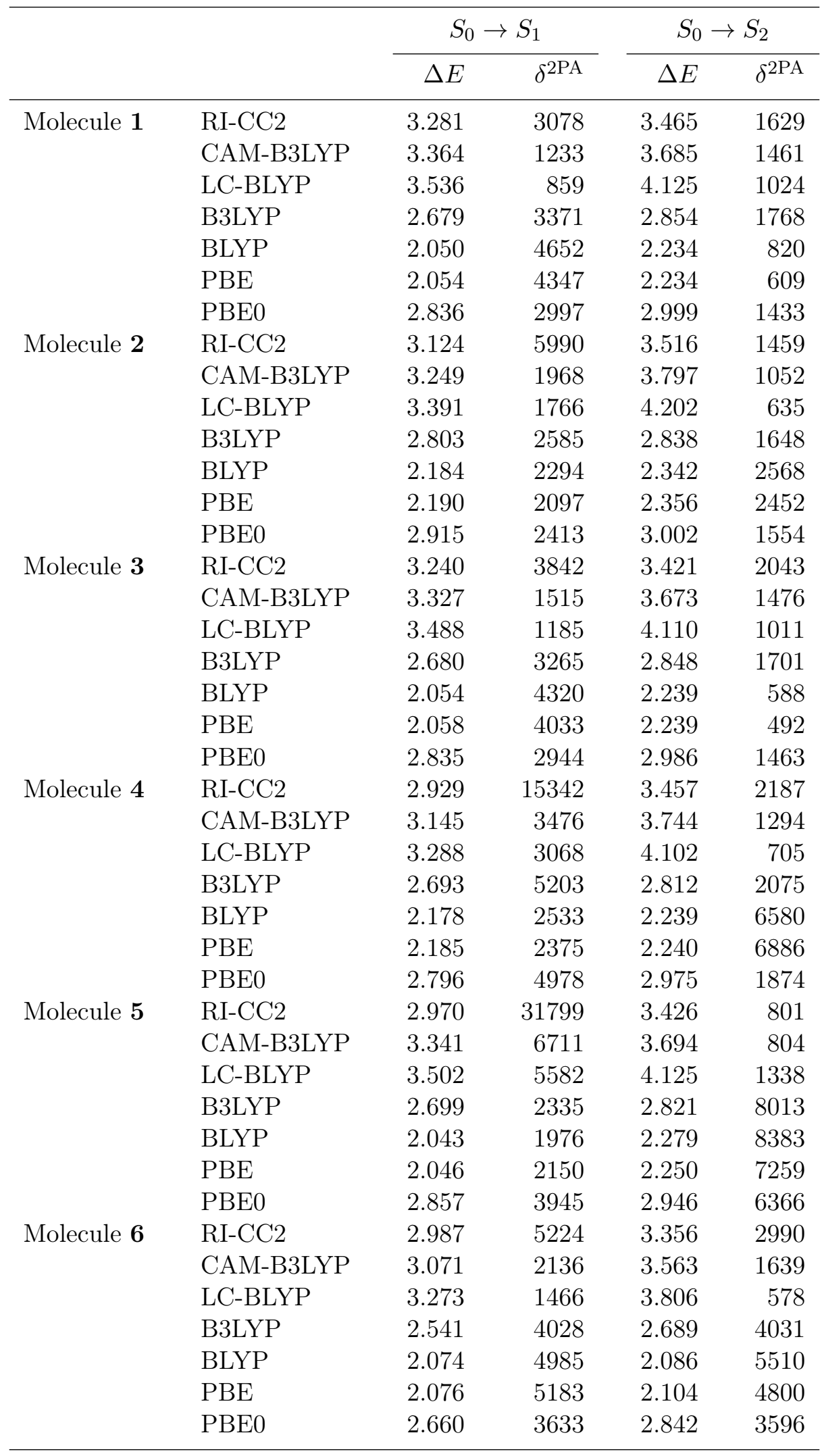


Table 2: Summary of statistical data analysis for two-photon absorption strengths, $\delta^{2 \mathrm{PA}}$. The ratio $\xi$ corresponds to $\xi=\delta_{\mathrm{CC} 2}^{2 \mathrm{PA}} / \delta_{\mathrm{DFT}}^{2 \mathrm{PA}}$. The data below encompass also molecule 5 in the case of PBE0 and the range-separated functionals. Values for $\xi$ for all molecules are given in the Supporting Information.

\begin{tabular}{|c|c|c|c|c|c|c|}
\hline & CAM-B3LYP & LC-BLYP & B3LYP & BLYP & PBE & PBE0 \\
\hline$\xi_{\text {ave }}$ & 3.28 & 4.08 & 1.73 & 1.43 & 1.48 & 2.90 \\
\hline$\xi_{\min }$ & 2.45 & 3.24 & 0.91 & 0.66 & 0.71 & 1.03 \\
\hline$\xi_{\max }$ & 4.74 & 5.70 & 2.95 & 2.33 & 2.44 & 8.06 \\
\hline
\end{tabular}


Graphical TOC Entry

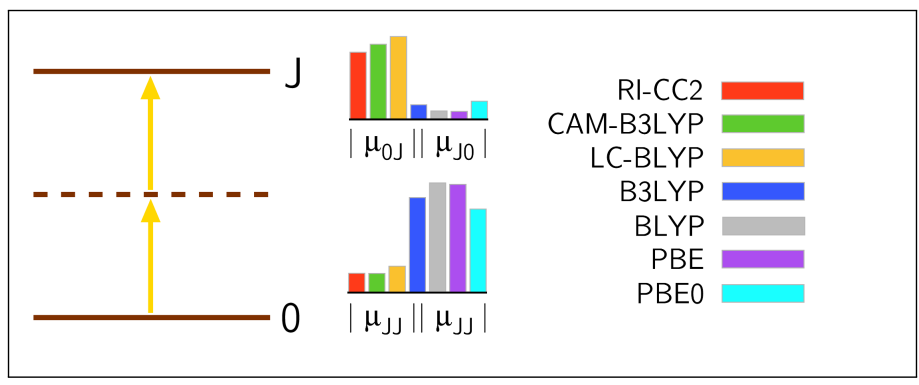

